\title{
Une nouvelle vision de l'assemblage du VIH-1
}

> Lors des étapes tardives de la réplication virale, les composants du virus sont adressés en un site cellulaire où ils s'assemblent pour donner des particules virales qui sont libérées dans le milieu extérieur. Ces étapes font actuellement l'objet d'un réexamen approfondi, à la lumière des développements technologiques récents en biologie moléculaire et cellulaire, et particulièrement en imagerie cellulaire. Dans le cas du virus de l'immunodéficience humaine de type 1 (VIH-1), une vision nouvelle de l'assemblage et de la production des particules, d'une complexité jusqu'alors insoupçonnée, s’en dégage. Des interactions spécifiques ont été identifiées entre composants viraux, et avec des facteurs cellulaires impliqués dans l'adressage et le bourgeonnement. À la multiplicité des signaux d'adressage correspond une variété de sites d'assemblage, dont le choix reste mal compris. Dans certaines cellules, cibles physiologiques de l'infection, les particules virales s'assembleraient et/ou s'accumuleraient dans des compartiments intracellulaires tels que les endosomes tardifs, pour être libérées dans le milieu extracellulaire, ou transmises directement à une cellule saine via une «synapse virale». Une telle vision « à couvert» de l'assemblage du VIH-l permet de mieux comprendre les stratégies virales d'infection, et sans doute de tracer de nouvelles approches thérapeutiques anti-VIH. <

\section{Le rôle pilote de la polyprotéine Gag}

Les particules rétrovirales sont constituées d'une enveloppe lipidique où sont ancrées les glycoprotéines virales Env, contenant le cœur viral. Celui-ci est composé de protéines et du génome viral constitué de deux molécules
Antoine Corbin, Boyan Grigorov, Philippe Roingeard, Jean-Luc Darlix, Delphine Muriaux

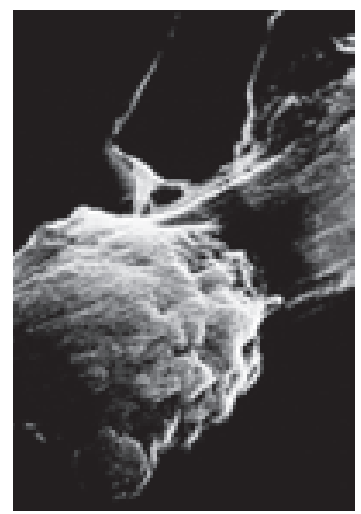

A. Corbin, B. Grigorov,

J.L. Darlix, D. Muriaux :

Laborétro

Unité de virologie humaine, Inserm U758, IFR128, École

Normale Supérieure de Lyon, 46, allée d'Italie, 69364 Lyon, France.

P. Roingeard : Inserm ERI 19, IFR

136, Faculté de Médecine, Université François Rabelais, 10, boulevard Tonnellé, BP 3223, 37032 Tours Cedex I, France. jldarlix@ens-lyon.fr

d'ARN simple-brin identiques appariées par des liaisons non-covalentes. Les protéines du cœur, dont la nature et la fonction sont décrites dans la Figure 1, sont produites par traduction de l'ARN génomique viral (ARNg) par des mécanismes complexes dont certains dépendent de sites d'entrée interne des ribosomes (IRES) [1], pour donner deux précurseurs polyprotéiques: Gag regroupe les protéines organisant la structure de la particule, et Gag-Pol contient les protéines à activité enzymatique (protéase, transcriptase inverse et intégrase), en plus des précédentes. Dans la plupart des rétrovirus, dont le VIH-1, l'assemblage du coeur se fait de façon concomitante au bourgeonnement viral et à la maturation protéolytique de Gag et Gag-Pol par la protéase virale, pour donner un virion enveloppé contenant les protéines matures (Figure 1).

\section{Les domaines de Gag}

Le précurseur Gag joue un rôle central dans ce processus : sa seule expression suffit pour produire des pseudo-particules virales (VLP) [2]. Dans les cellules infectées, la formation de virus est pilotée par l'association de Gag et de Gag-Pol avec des membranes cellulaires et leur multimérisation, lors du recrutement des deux molécules d'ARNg, de la protéine Env et de facteurs cellulaires facilitant le bourgeonnement. Moins d'une dizaine de spicules, formés d'un trimère d'Env, sont observés sur 
les virions infectieux, qui contiennent environ 1500 copies de Gag [3]. Dans le cas du VIH-1, des protéines régulatrices telles que Vpr sont aussi incorporées via des interactions spécifiques avec Gag [4]. La Figure IB illustre les déterminants majeurs de Gag qui interviennent dans ces différents mécanismes.
Plusieurs domaines sont impliqués dans des interactions Gag-Gag nécessaires à l'assemblage des particules. La capside (CA) joue un rôle majeur dans la cohésion mécanique des virions et l'arrangement multimérique de Gag. Elle est constituée de deux domaines amino- et carboxy-

Figure 1. Le précurseur Gag et l'assemblage du VIH-1. A. Schéma d'un virus mature. Les particules virales sont constituées d'une enveloppe lipidique dans laquelle sont insérés des spicules constitués de trimères de glycoprotéine d'enveloppe ( $\varepsilon$ nv), elle même formée des sous-unités de surface (SU) et transmembranaire (TM). Au cours du bourgeonnement, les précurseurs polyprotéiques Gag et Gag-Pol sont clivés par la protéase virale pour donner les protéines virales matures: matrice (MA), capside (CA), nucléocapside (NC), protéase (PR), transcriptase inverse (RT), intégrase (IN), et trois peptides : p6, SP1 et SP2 (voir en B). MA reste associée à la face interne de l'enveloppe. CA forme le cœur conique, contenant le génome viral formé d'un dimère d'ARN génomique (ARNg) recouvert de molécules NC, des ARN de transfert cellulaires nécessaires à l'initiation de sa réplication, ainsi que les enzymes codées par Pol (PR, RT et IN) et des protéines régulatrices associées à Gag, comme Vpr. RT et IN sont respectivement nécessaires à la transcription inverse du génome et à son intégration dans le génome cellulaire. B. Le précurseur protéique Gag joue un rôle central dans l'assemblage viral. Sa seule expression dans les cellules suffit à produire des pseudo-particules virales (VLP). L'assemblage de Gag nécessite deux plateformes constituées par l'ARNg et par une membrane cellulaire, probablement au niveau de microdomaines membranaires tels que les radeaux lipidiques. L'interaction de l'ARNg avec une séquence chargée positivement (+++), portée par le domaine NC, favorise la multimérisation

A

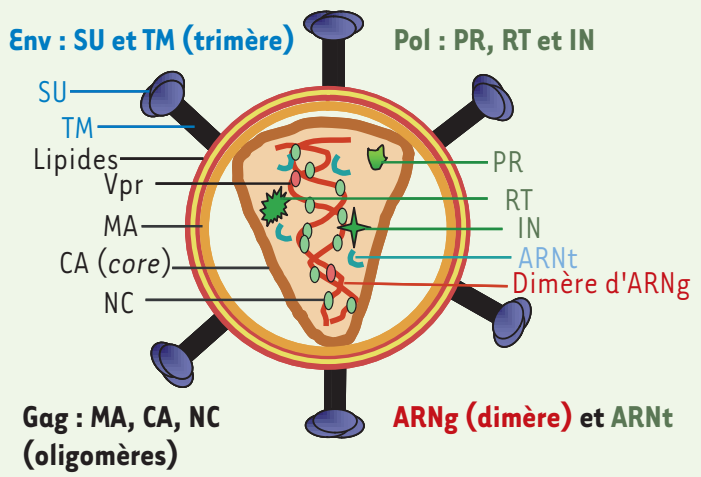

de Gag, stabilisée par le domaine CA. NC, via ses doigts à zinc, est responsable de la spécificité d'incorporation de l'ARNg et joue un rôle de protéine chaperone des acides nucléiques viraux nécessaire tout au long du cycle viral. Au sein de ce complexe, Gag expose un résidu myristate amino-terminal et permet son interaction avec les bicouches lipidiques, stabilisée par l'interaction d'une région amino-terminale du domaine $M A$, chargée positivement $(+++)$, avec les phospholipides membranaires. Le domaine MA présente aussi de nombreux signaux nécessaires à l'adressage de Gag à diverses membranes cellulaires, comme les sites d'interactions avec les protéines adaptatrices de la clathrine AP-2 et AP-3, ou l'interaction avec la glycoprotéine d'enveloppe virale. Enfin, p6 correspond à un «domaine tardif », dont l'interaction avec des membres des complexes cellulaires ESCRT (Tsg101, AIP-1) est requise lors des phases tardives du bourgeonnement et de la libération des particules virales. NC et p6 interviennent aussi dans l'incorporation de Vpr dans les particules virales.

\section{B}

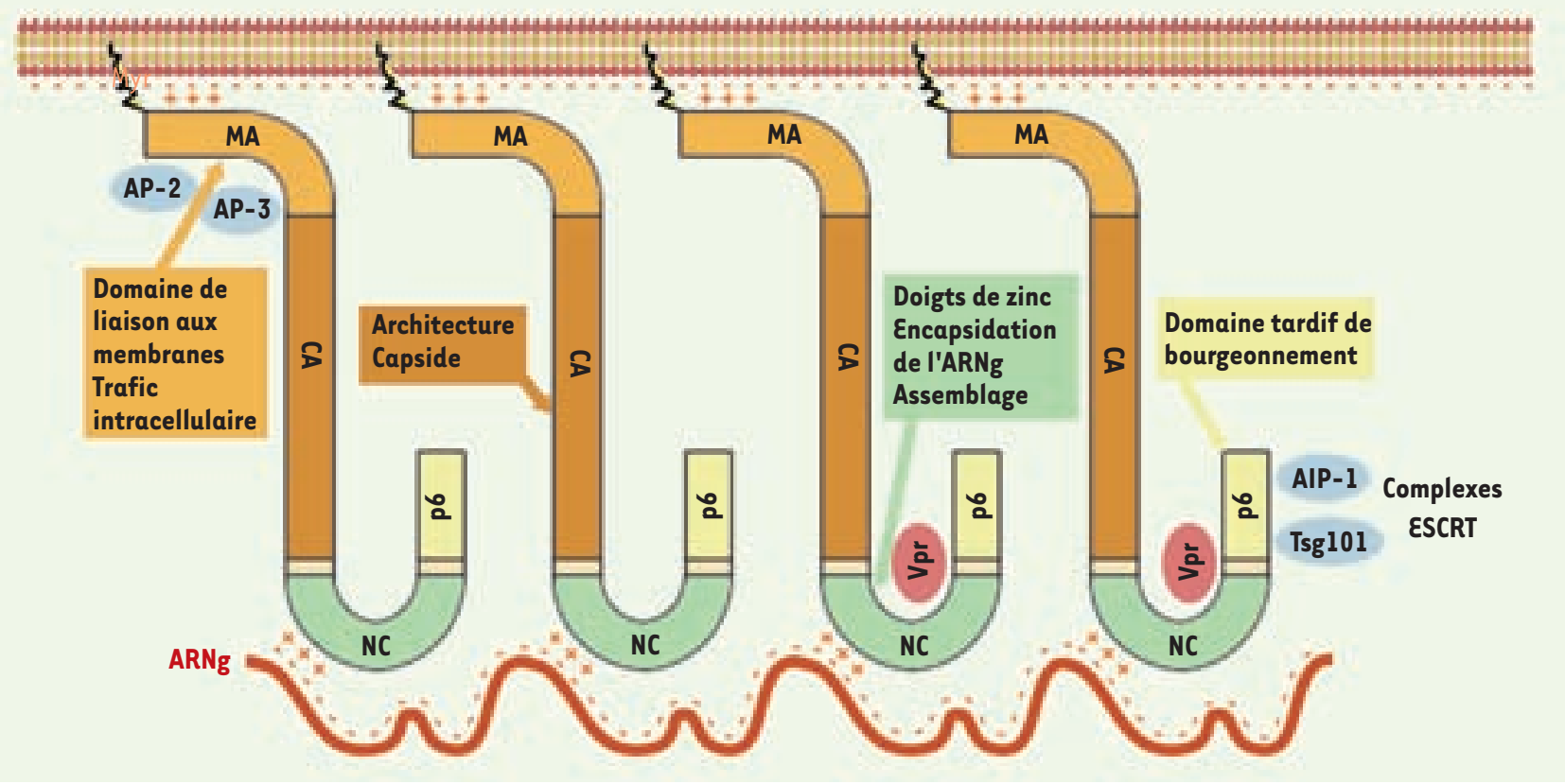


terminaux (NTD et CTD). Dans des expériences d'assemblage in vitro, le CTD, qui contient le motif le mieux conservé de Gag (Major Homology Region), forme un dimère stable. Le NTD forme alors des hexamères s'associant en réseau par le biais des dimères de CTD [5]. Dans Gag, I'hélice carboxy-terminale du CTD se prolonge dans un court peptide (SP1) libéré après maturation par la protéase et reliant CA à la nucléocapside (NC). SPl et la partie amino-terminale de la NC sont nécessaires à la multimérisation de Gag. Cette dernière, essentiellement peu structurée, contient plusieurs acides aminés basiques dont l'importance pour cette fonction est strictement corrélée à leur rôle dans l'interaction de Gag avec les ARN et leur incorporation dans les virions [6], suggérant que I'ARN jouerait un rôle dans la multimérisation de Gag. De plus, la partie centrale de la NC, présentant une structure globulaire formée de deux doigts à zinc, est indispensable au recrutement spécifique de l'ARNg, et à l'assemblage de virions infectieux [7].

Deux déterminants du domaine amino-terminal (MA) de Gag du VIH-1, conservés chez les rétrovirus, jouent un rôle majeur dans son association aux membranes. La partie amino-terminale de MA forme un domaine globulaire constitué de 4 hélices, à la surface duquel sont regroupés une série d'acides aminés basiques qui interagissent avec les phospholipides membranaires, et plus spécifiquement avec un phosphatidyl-inositol phosphate (PIP), le PI $(4,5)$ P2 [8, 9], un marqueur de la face cytosolique de la membrane plasmique, ciblant ainsi l'expression de Gag à la surface de la cellule. Par ailleurs, MA présente un site de myristylation à son extrémité amino-terminale, nécessaire à l'association stable aux membranes cellulaires et à la formation de particules virales. Deux structures alternatives de MA du VIH-l ont été publiées, dans lesquelles le myristate est soit enfoui dans la structure protéique, soit exposé à sa surface. Son exposition serait déclenchée par l'oligomérisation de Gag et son interaction avec le $\mathrm{PI}(4,5) \mathrm{P} 2$ [10], et inhibée par sa maturation protéolytique [11], suggérant que la stabilité de l'association de Gag avec les membranes pourrait être renforcée transitoirement au moment de l'assemblage et du bourgeonnement, et revenir au niveau basal après maturation de Gag par la protéase virale.

Ces données suggèrent un modèle dans lequel l'assemblage de Gag est guidé par une double plateforme constituée de l'ARNg, dont l'interaction spécifique avec NC permet la nucléation de l'assemblage et sa propre incorporation dans les virions, et d'une membrane cellulaire sur laquelle les molécules se concentrent et s'alignent sous l'influence de MA.

De surcroît, des mutations du domaine carboxy-terminal de Gag (p6), riche en prolines, ont montré l'importance de son interaction avec des facteurs cellulaires lors du bourgeonnement et de la libération des virions. Ainsi, un motif PTAP, qui est un déterminant de cette fonction, interagit avec Tsg101. Un deuxième motif de p6 interagit avec AIP-1/ Alix, et un peptide correspondant au domaine de liaison de AIP-1/Alix à la p6 de VIH-1 constitue un inhibiteur puissant de la libération des virions $[12,13]$. Des «domaines tardifs » riches en prolines, présentant des motifs similaires, interagissant avec les mêmes acteurs cellulaires et importants pour les étapes tardives du bourgeonnement viral, ont été trouvés à des positions variables de Gag dans d'autres rétrovirus, et dans les protéines de capside de plusieurs autres familles de virus à ARN enveloppés [14].
Homologies du processus de bourgeonnement viral avec la biogenèse des endosomes

Ces données ont eu un retentissement important du fait de leur caractère général, et parce qu'elles établissent un lien entre le bourgeonnement viral et le seul phénomène cellulaire topologiquement équivalent: le bourgeonnement d'une région cytosolique à travers une membrane, pour aboutir à la libération de vésicules. En effet, Tsg101 et AIP-1/Alix font partie des Class $\varepsilon$ vesicular protein sorting $(V p s-\varepsilon)$, une classe de protéines impliquées dans la biogenèse des endosomes tardifs ou multivesicular bodies (MVB) et le tri des molécules «cargo» à leur niveau. Le bourgeonnement dans la lumière des MVB de domaines membranaires concentrant ces molécules cargo aboutit à la formation de vésicules internes [15], généralement dégradées après fusion avec les lysosomes. Elles peuvent aussi, dans certains types cellulaires, être recyclées après fusion avec la membrane plasmique, ou être relarguées dans le milieu extérieur sous forme $d^{\prime}$ « exosomes » [16]. Les Vps- $\varepsilon$ sont des protéines cytosoliques dont les fonctions restent mal comprises, et dont la plupart sont regroupées au sein de complexes dénommés ESCRT (endosomal sorting complex required for transport) [17].

La reconnaissance de la membrane endosomale par l'ESCRT-0/Hrs aboutit au recrutement successif des ESCRT-I, ESCRT-II puis ESCRT-III. Ce dernier s'assemble en un complexe de haut poids moléculaire qui intervient dans la formation des vésicules internes, et dont le désassemblage ATP-dépendant par la protéine Vps-4 est nécessaire au bourgeonnement. Le ciblage des molécules cargo vers les vésicules internes dépend principalement de leur mono-ubiquitinylation et fait intervenir les complexes ESCRT, dont plusieurs membres possèdent des domaines de reconnaissance de l'ubiquitine.

Gag possède plusieurs sites de mono-ubiquitinylation [18], et certains d'entre eux - mais pas celui du VIH-1 ont dans leur domaine tardif un motif PPxy, recrutant une ubiquitine-ligase $\varepsilon 3$ de type Nedd-4. Les fonctions potentielles de ces deux déterminants suggèrent l'importance de la mono-ubiquitinylation pour le bourgeonnement viral. Les rôles de Tsgl0l et de AIP-1/Alix restent cependant imprécis. Tsg101 est un membre d'ESCRT-I, ayant un site de liaison à l'ubiquitine, et pourrait intervenir dans le tri des molécules cargo. AIP1/Alix est une protéine multifonctionnelle qui interagit avec des membres de ESCRT-III, mais aussi avec Tsg101, par un motif PTAP similaire à celui du VIH-1.

Enfin, ISG15 - une protéine induite par l'interféron inhibe l'ubiquitinylation de Gag et de Tsg101, l'interaction Gag/Tsgl01 et le bourgeonnement viral [19], cette étape du cycle viral constituant une cible privilégiée 
des systèmes de défenses intracellulaires contre les rétrovirus induits par l'interféron.

\section{Le trafic cellulaire des constituants viraux}

Replacer ces mécanismes d'assemblage dans leur contexte cellulaire constitue un enjeu important. Les voies de trafic empruntées par Gag, Env et l'ARNg jusqu'au site d'assemblage (décrites dans la Figure 2) et leurs modalités d'interaction restent mal documentées et fort débattues. Ainsi, Gag présente de multiples signaux d'adressage vers différents compartiments membranaires. L'interaction de MA avec le PI $(4,5) \mathrm{P} 2$ (voir plus haut) cible Gag à la membrane plasmique, et la déplétion de ce lipide dans les cellules le redirige vers les endosomes tardifs [8]. Inversement, un site d'interaction avec $\mathrm{AP}-3$, situé dans la même région, dirigerait

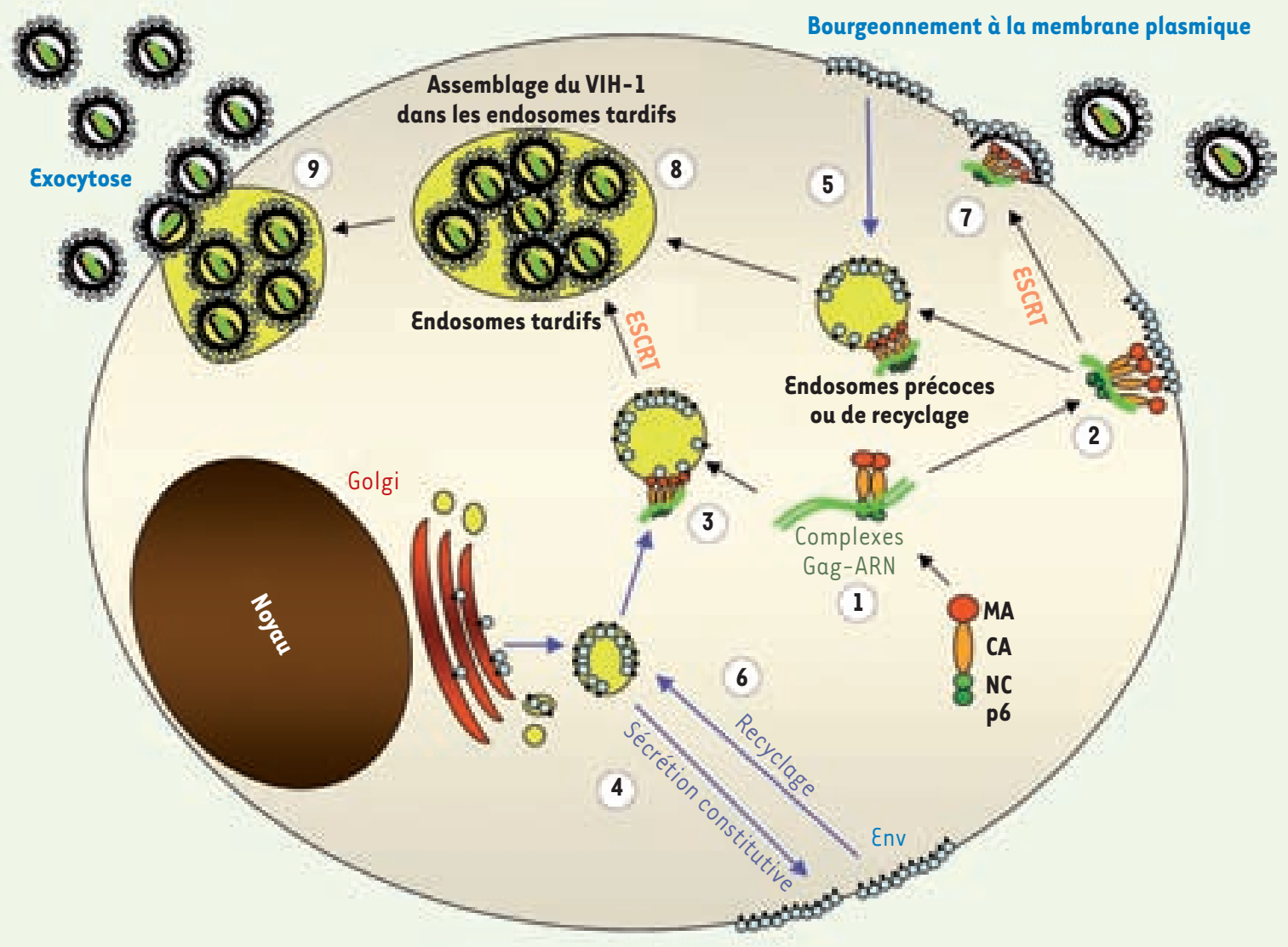

Figure 2. Trafic et voies d'assemblage du VIH-1. Lors des phases tardives de la réplication virale, les molécules de Gag sont synthétisées au niveau des polysomes, sans doute dans le cytoplasme et se multimérisent sur l'ARN génomique viral (ARNg) grâce au domaine nucléocapside (NC) (1), formant ainsi des complexes ribonucléoprotéiques (RNP) viraux qui s'ancrent aux membranes de la cellule infectée. Sous l'effet de multiples signaux d'adressage au sein de Gag, les complexes peuvent être dirigés vers la membrane plasmique (2) ou des compartiments endosomaux (3). La formation de virus infectieux nécessite le recrutement de la glycoprotéine d'enveloppe (Env) au niveau de ces complexes. L'enveloppe est produite dans le réticulum endoplasmique, glycosylée dans le Golgi et secrétée jusqu'à la membrane plasmique (4) d'où elle est recyclée. Elle peut se retrouver dans les endosomes précoces, de recyclage, et tardifs (5), ou suivre un transport rétrograde des endosomes tardifs vers le réseau trans-golgien (6). Une interaction précoce de l'Env avec les complexes Gag/ARNg pourrait aussi influer sur le trafic de ceux-ci. Le choix de la membrane d'assemblage viral dépend du type cellulaire et pourrait résulter d'un jeu complexe entre signaux d'adressage. Par exemple, dans les cellules cancéreuses 293T, l'assemblage s'observe à la fois à la membrane plasmique (7) et dans les endosomes (8). Dans les lymphocytes T CD4 ${ }^{+}$ chroniquement infectés par VIH-1 et dans les macrophages, le virus s'assemble dans les endosomes tardifs (8). Dans les deux cas, Gag recrute les complexes ESCRT nécessaires aux phases tardives du bourgeonnement [13]. L'accumulation de virions matures dans les endosomes tardifs montre que le VIH-l exploite cette voie de trafic intracellulaire à son avantage. Sous l'effet d'une stimulation cellulaire, ces virions seraient exocytés par fusion des endosomes tardifs avec la membrane plasmique et relargués massivement dans le milieu extracellulaire ou transférés spécifiquement à une autre cellule au niveau de points de contact intercellulaires (9). 
Gag depuis le réseau trans-Golgien vers les endosomes tardifs [20], et un site d'interaction avec AP-2 à la jonction MA-CA permettrait son internalisation de la membrane plasmique vers le compartiment endosomal. L'étape à laquelle a lieu le recrutement de l'ARNg sous la dépendance du domaine NC [21], qui influence la polymérisation de Gag et son interaction avec les bicouches lipidiques, demeure mal définie, tout comme le rôle du cytosquelette dans ces transports [22]. Env semble rejoindre la membrane plasmique par les voies de sécrétion, et présente dans son domaine cytosolique des signaux redondants qui assureraient son endocytose par des voies dépendantes d'AP-2 [23], mais aussi son recyclage des endosomes vers le réseau trans-golgien, par un domaine d'interaction avec le facteur cellulaire TIP47 [24], spécifique de cette fonction. Elle pourrait alors influencer le trafic de Gag, comme le suggère l'observation que l'expression de $\varepsilon n v$ restreint le bourgeonnement viral au pôle basolatéral dans des cellules polarisées [25]. Les modalités d'interaction Env-Gag sont fort débattues. Le remplacement de Env par certaines enveloppes virales non apparentées donne des virions infectieux, ce qui plaide pour un mécanisme passif, tel que la co-localisation de Env et Gag au niveau de microdomaines membranaires correspondant au site d'assemblage (voir plus loin). Pourtant, des mutations de la queue cytoplasmique de Env ou du domaine MA de Gag affectent l'efficacité d'incorporation de Env dans les virions [26], sans qu'une interaction directe ait pu être détectée. Récemment, un site de reconnaissance de TIP47 a été identifié dans le domaine amino-terminal de MA, permettant un pontage entre Gag et Env et nécessaire au recrutement de Env [27]. Ainsi TIP47 pourrait, en l'absence de Gag, positionner Env au niveau du réseau trans-golgien, puis, en sa présence, induire l'interaction Gag-Env et le ciblage du complexe sous le contrôle de Env.

\section{Où et comment bourgeonne le virus ?}

L'ensemble de ces données s'accordent sur le rôle central de l'exploitation du trafic vésiculaire par le virus et de l'interaction avec les facteurs cellulaires qui y sont associés, mais leur caractère contradictoire empêche toute conclusion quant à la localisation cellulaire de l'assemblage. Si le bourgeonnement du VIH-l était classiquement décrit à la membrane plasmique, il avait aussi été observé dans des macrophages au niveau de compartiments intracellulaires [28] apparentés aux MVB [29].

\section{Une variété de sites possibles de bourgeonnement}

La découverte de l'interaction entre les domaines tardifs et les complexes ESCRT a conduit à un regain d'intérêt pour ces observations et une polémique s'est engagée sur le site réel du bourgeonnement viral, certains arguant que les virions endosomaux proviennent de l'endocytose de virus formés à la membrane plasmique [30], d'autres au contraire que ceux vus à la membrane plasmique proviennent de la fusion des endosomes tardifs avec cette dernière. Plus récemment, il a été suggéré que les compartiments intracellulaires observés pourraient correspondre en fait à des domaines de la membrane plasmique formant de profondes invaginations dans la cellule [31, 32].
L'implication de la machinerie de biogenèse des MVB milite évidemment pour un bourgeonnement endosomique. Toutefois, I'interaction de Gag avec Tsgl0l et AIP1/Alix permettrait le recrutement des complexes ESCRT à la membrane plasmique en court-circuitant le complexe ESCRT-0/Hrs, responsable du ciblage aux endosomes. Le recrutement de Tsg101 par Gag a d'ailleurs été observé à la membrane plasmique [33].

La composition des membranes cellulaires varie en fonction de leur nature (membrane plasmique, voies de sécrétion ou d'endocytose...) et de microdomaines où se regroupent certains constituants, qui ont surtout été décrits dans le cas de la membrane plasmique et dont l'hétérogénéité, l'origine et la signification précises restent mal comprises. L'enveloppe du VIH-1 est fortement enrichie en sphingomyéline et en cholestérol [34]. Cette composition, sensée refléter celle de son lieu de bourgeonnement, confère aux domaines membranaires correspondants des propriétés d'insolubilité en présence de détergents (detergent-resistant microdomains, DRM). Gag se concentre dans les DRM et une déplétion en cholestérol, qui inhibe leur formation, diminue la production de virions. Elle reflète assez bien celle de microdomaines de la membrane plasmique, les radeaux lipidiques, tout en étant proche de celle des endosomes tardifs. De même, l'incorporation de protéines membranaires dans les virions reflète, selon les cas, la composition des radeaux lipidiques ou celle des MVB [35].

Déterminants du site de bourgeonnement : rôle du caractère aigu ou chronique de l'infection

Au total, un quasi-consensus semble s'établir sur le fait que les deux sites de bourgeonnement peuvent coexister. La question est alors de comprendre les mécanismes et les raisons pour lesquels l'un ou l'autre site est privilégié.

Malgré son caractère déroutant, la multiplicité des signaux d'adressage pourrait expliquer le caractère contradictoire des résultats publiés. La mutation d'un signal conduit souvent à démasquer l'effet d'un signal opposé $[8,20]$. Le caractère chevauchant de beaucoup de ces signaux est à ce propos significatif. Ainsi, le domaine amino-terminal de MA est responsable de multiples interactions de Gag : avec les phospholipides membranaires impliqués dans l'arrimage aux bicouches lipidiques, le PI $(4,5) P 2$ (assurant un ciblage à la membrane plasmique [10]), AP-3 (le ciblant au contraire vers les MVB [20]), et TIP47, impliqué dans le recrutement de Env [27]. On peut difficilement envisager que ces interactions soient simultanées. Elles pourraient se succéder pour assurer un déroulement harmonieux 
des étapes tardives du cycle viral, et conféreraient aussi une grande plasticité fonctionnelle au virus, par exemple pour le choix du site d'assemblage, qui résulterait de l'équilibre entre ces signaux, différent selon le système étudié.

En effet, le bourgeonnement est majoritairement observé au niveau des MVB dans les macrophages $[28,36]$, et de la membrane plasmique dans les lymphocytes et les cellules épithéliales. Toutefois, des résultats récents de notre laboratoire indiquent que le type cellulaire n'est pas le déterminant majeur, puisque dans une lignée lymphocytaire chroniquement infectée, le bourgeonnement dans les MVB est prédominant [37] (Figure 3). Ce résultat, réminiscent de l'observation initiale faite sur une lignée macrophagique [28], suggère que dans les cellules chroniquement infectées, le bourgeonnement serait restreint aux MVB, alors que le bourgeonnement à la membrane plasmique serait associé au caractère aigu des infections, caractérisé par une production virale massive, des effets cytopathiques importants et une forte mortalité cellulaire. Cette production massive de virus pourrait entraîner un débordement des capacités d'assemblage des MVB vers la membrane plasmique. Les macrophages et lymphocytes chroniquement infectés constituent les réservoirs viraux impliqués in vivo dans la persistance à long terme de l'infection, y compris chez les personnes traitées par les polythérapies antirétrovirales (HAART). L'assemblage et l'accumulation des virions dans les MVB permettraient à ces cellules d'échapper au système immunitaire, du fait de l'absence d'expression membranaire des constituants viraux, tout en relarguant massivement des virions dans des circonstances privilégiées [38, 37]. L'infection par les rétrovirus, y compris le VIH-1, se fait préférentiellement lors de contacts directs entre cellules. Les mécanismes mis en jeu, décrits sous le terme de «synapse virologique» [39], réminiscents de la synapse immunologique dont ils empruntent certains composants
[40], font intervenir des remaniements du cytosquelette qui aboutissent à la formation de «filopodes » au bout desquels s'établissent des zones d'adhésion entre cellules constituant des sites privilégiés de transport de virus [41]. La mise en place de ces synapses virologiques pourrait être le déclencheur d'une libération polarisée des virions à partir des réservoirs endosomiques et permettre ainsi la dissémination du VIH-l de cellules à cellules, processus efficace et peu accessible aux défenses immunitaires.

Des données similaires ont été obtenues pour le trafic, l'assemblage et le bourgeonnement d'autres rétrovirus. Par exemple, l'infection se fait préférentiellement par contact cellulaire pour la plupart d'entre eux [42]. Ainsi les grandes lignes de ce modèle pourraient être générales chez les rétrovirus, y compris pour d'autres pathogènes humains. $\diamond$

\section{REMERCIEMENTS}

Les travaux du laboratoire bénéficient d'un soutien de l'Anrs, de l'Inserm, du CNRS, de Europe (TRIOH) et de Sidaction.

\section{SUMMARY}

\section{Revisiting HIV-1 assembly}

During the late stage of virus replication, incorporation of the envelope glycoproteins (Env) by Gag cores takes place together with the proteolytic maturation of Gag and Gag-Pol precursors. Assembly is initially driven by Gag oligomerisation, which requires two platorms. The first one is formed by specific membrane subdomains

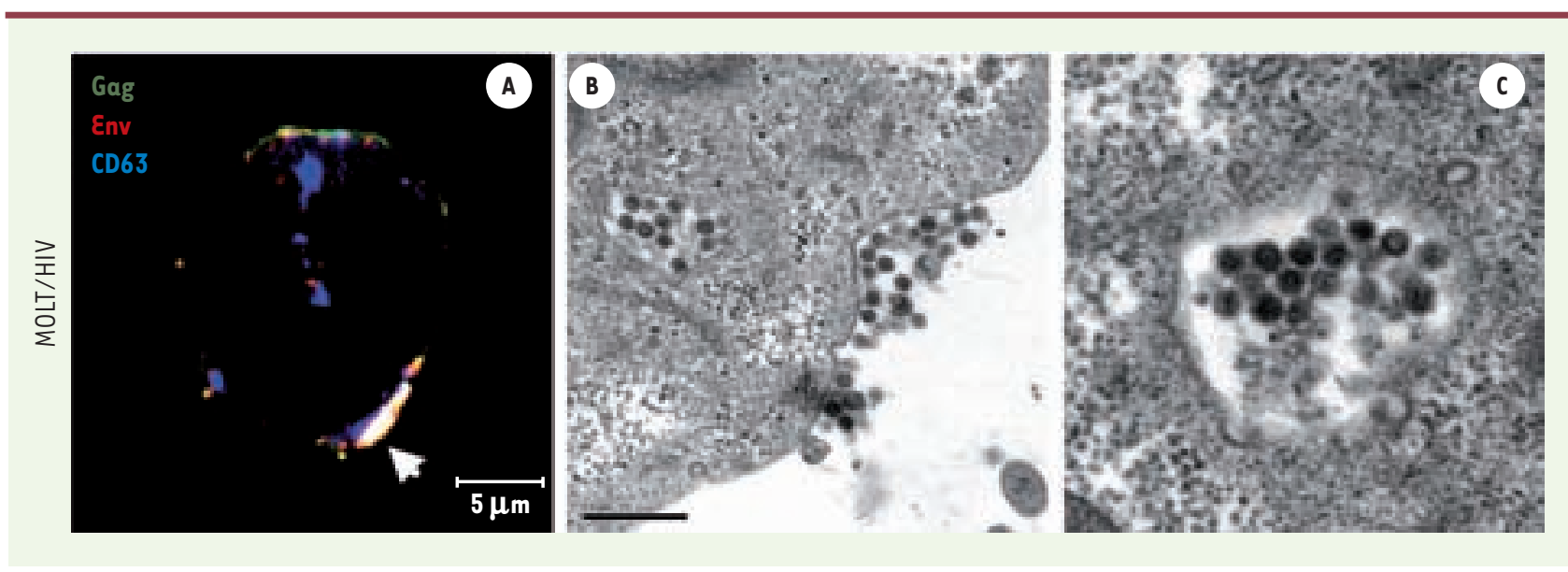

Figure 3. Accumulation des virions VIH-1 dans les endosomes tardifs de cellules lymphoblastiques T chroniquement infectées par VIH-1. A. Observation par microscopie confocale de la localisation intracellulaire des protéines Gag et Env. L'immuno-détection a été faite par des anticorps dirigés contre Gag (vert), Env (rouge) et CD63, un marqueur des endosomes tardifs (bleu). Des triples marquages (en blanc), correspondant à une co-localisation de Gag et Env dans des endosomes tardifs, sont visualisés dans un compartiment de grande taille, proche de la surface cellulaire (flèche blanche). B, C. Observation par microscopie électronique des cellules, montrant l'accumulation de particules virales dans des vésicules intracellulaires ( $B$ et $C$ ) et leur libération à la membrane plasmique (B). 
with which Gag molecules interact via the N-terminal MA domain, and the second by the viral genomic RNA undergoing specific interactions with the NC domain of Gag. To complete viral budding, the Gag «late domain » subsequently associates with members of the ESCRT complexes involved in the budding of vesicles in late endosomes $(L E)$. While the cellular trafficking of the viral components is still poorly understood, there is an ongoing debate on the site of HIV-1 assembly, because this process might take place either at the plasma membrane or in intracellular compartments such as the $L \mathcal{L}$, depending on the virus/cell system studied. This site may depend on the interplay of multiple overlapping trafficking signals bear by Gag and Env. Our recent results indicate that it may rely on the chronic or acute nature of the viral infection more than on the cell type. In chronically infected cells, virions probably assemble and accumulate in intracellular compartments hidden from the immune system. Release of virions in the form of bursts would be triggered during cell-cell interactions, through a specialized structure called the virological synapse. $\diamond$

\section{RÉFÉRENCES}

1. Balvay L, Lopez Lastra M, Sargueil B, et al. Translational control of retroviruses. Nat Rev Microbiol 2007 ; 5 : 128-40.

2. Muriaux D, Mirro J, Harvin D, Rein A. RNA is a structural element in retrovirus particles. Proc Natl Acad Sci USA 2001 ; 98 : 5246-51.

3. Gatignol A, Dubuisson J, Wainberg MA, et al. New pandemics : HIV and AIDS, HCV and chronic hepatitis, influenza virus and flu. Retrovirology $2007 ; 4: 8$.

4. De Rocquigny H, Petitjean P, Tanchou V, et al. The zinc fingers of HIV nucleocapsid protein $\mathrm{NCp} 7$ direct interactions with the viral regulatory protein Vpr. J Biol Chem 1997; 272 : 30753-9.

5. Huseby D, Barklis RL, Alfadhli A, Barklis $\varepsilon$. Assembly of human immunodeficiency virus precursor gag proteins. J Biol Chem 2005; $280: 17664-70$.

6. Cimarelli A, Sandin S, Höglund S, Luban J. Basic residues in human immunodeficiency virus type 1 nucleocapsid promote virion assembly via interaction with RNA.J Virol $2000 ; 74: 3046-57$.

7. Ottmann M, Gabus C, Darlix JL. The central globular domain of the nucleocapsid protein of human immunodeficiency virus type 1 is critical for virion structure and infectivity. J Virol $1995 ; 69$ : 1778-84.

8. Ono A, Ablan SD, Lockett SJ, et al. Phosphatidylinositol $(4,5)$ bisphosphate regulates HIV-l Gag targeting to the plasma membrane. Proc Natl Acad Sci USA 2004 ; 101 : 14889-94.

9. Saad JS, Miller J, Tai J, et al. Structural basis for targeting HIV-1 Gag proteins to the plasma membrane for virus assembly. Proc Natl Acad Sci USA $2006 ; 103: 11364-9$.

10. Saad JS, Loeliger $\varepsilon$, Luncsford P, et al. Point mutations in the HIV-1 matrix protein turn off the myristyl switch. J Mol Biol $2007 ; 366$ : 574-85.

11. Spearman P, Horton R, Ratner L, Kuli-Zade I. Membrane binding of human immunodeficiency virus type 1 matrix protein in vivo supports a conformational myristyl switch mechanism. J Virol 1997; $71: 6582-92$.

12. Demirov DG, Freed $\varepsilon 0$. Retrovirus budding. Virus Res 2004 ; $106: 87-102$.

13. Goff SP. Host factors exploited by retroviruses. Nat Rev Microbiol 2007 ; $5: 253-63$

14. Bieniasz PD. Late budding domains and host proteins in enveloped virus release. Virology $2006 ; 344: 55-63$.

15. Russell MR, Nickerson DP, Odorizzi G. Molecular mechanisms of late endosome morphology, identity and sorting. Curr Opin Cell Biol 2006; $18: 422-8$.
16. Février B, Raposo G. Exosomes : endosomal-derived vesicles shipping extracellular messages. Curr Opin Cell Biol $2004 ; 16: 415-21$.

17. Hurley JH, Emr SD. The ESCRT complexes : structure and mechanism of a membrane-trafficking network. Annu Rev Bioph Biomol Struct $2006 ; 35: 277-98$.

18. Gottwein $\varepsilon$, Jäger $S$, Habermann A, Kräusslich HG. Cumulative mutations of ubiquitin acceptor sites in human immunodeficiency virus type 1 gag cause a late budding defect. J Virol 2006; $80: 6267-75$

19. Okumura A, Lu G, Pitha-Rowe I, Pitha PM. Innate antiviral response targets HIV-1 release by the induction of ubiquitin-like protein ISG15. Proc Natl Acad Sci USA 2006 ; 103: 1440-5.

20. Dong X, Li H, Derdowski A, et al. AP-3 directs the intracellular trafficking of HIV-1 Gag and plays a key role in particle assembly. Cell $2005 ; 120: 663-74$.

21. Darlix JL, Lapadat-Tapolsky M, de Rocquigny H, Roques BP. First glimpses at structurefunction relationships of the nucleocapsid protein of retroviruses. J Mol Biol 1995; $254: 523-37$.

22. Fackler $\mathrm{OT}$, Kräusslich HG. Interactions of human retroviruses with the host cell cytoskeleton. Curr Opin Microbiol 2006; $9:$ 409-15.

23. Byland R, Vance PJ, Hoxie JA, Marsh M. A conserved dileucine motif mediates clathrin and AP2-dependent endocytosis of the HIV-1 envelope protein. Mol Biol Cell 2007 ; 18: 414-25.

24. Blot $G$, Janvier $K$, Le Panse $S$, et al. Targeting of the human immunodeficiency virus type 1 envelope to the trans-Golgi network through binding to TIP47 is required for env incorporation into virions and infectivity. J Virol $2003 ; 77: 6931-45$.

25. Owens RJ, Dubay JW, Hunter $\varepsilon$, Compans RW. Human immunodeficiency virus envelope protein determines the site of virus release in polarized epithelial cells. Proc Natl Acad Sci USA 1991 $88: 3987-91$

26. Murakami T, Freed $\varepsilon 0$. Genetic evidence for an interaction between human immunodeficiency virus type 1 matrix and alpha-helix 2 of the gp 41 cytoplasmic tail. J Virol $2000 ; 74: 3548-54$.

27. Lopez-Vergès $S$, Camus $G$, Blot $G$, et al. Tail-interacting protein TIP47 is a connector between Gag and Env and is required for Env incorporation into HIV-1 virions. Proc Natl Acad Sci USA $2006 ; 103: 14947-52$.

28. Orenstein JM, Meltzer MS, Phipps T, Gendelman HE. Cytoplasmic assembly and accumulation of human immunodeficiency virus types 1 and 2 in recombinant human colony-stimulating factor-1-treated human monocytes : an ultrastructural study. J Virol 1988; 62 : 2578-86.

29. Raposo G, Moore M, Innes D, et al. Human macrophages accumulate HIV-1 particles in MHC II compartments. Traffic $2002 ; 3: 718-29$.

30. Jouvenet $\mathrm{N}$, Neil SJ, Bess $\mathrm{C}$, et al. Plasma membrane is the site of productive HIV-1 particle assembly. PLoS Biol $2006 ; 4$ : e435.

31. Welsch S, Keppler OT, Habermann A, et al. HIV-1 buds predominantly at the plasma membrane of primary human macrophages. PLoS Pathog $2007 ; 3:$ e36.

32. Deneka M, Pelchen-Matthews A, Byland R et al. In macrophages, HIV-1 assembles into an intracellular plasma membrane domain containing the tetraspanins CD81, CD9, and CD53. J Cell Biol 2007 ; 177 : 329-41.

33. Derdowski A, Ding L, Spearman P. A novel fluorescence resonance energy transfer assay demonstrates that the human immunodeficiency virus type 1 Pr55Gag I domain mediates Gag-Gag interactions.J Virol $2004 ; 78: 1230-42$.

34. Brügger $B, G$ lass $B$, Haberkant $P$, et al. The HIV lipidome : a raft with an unusual composition. Proc Natl Acad Sci USA $2006 ; 103: 2641-6$.

35. Nydegger $S$, Khurana $S$, Krementsov DN, et al. Mapping of tetraspanin-enriched microdomains that can function as gateways for HIV-1.J Cell Biol $2006 ; 173$ : 795-807.

36. Pelchen-Matthews A, Kramer B, Marsh M. Infectious HIV-1 assembles in late endosomes in primary macrophages. J Cell Biol 2003; 162: 443-55.

37. Grigorov B, Arcanger F, Roingeard P, et al. Assembly of infectious HIV-1 in human epithelial and T-lymphoblastic cell lines. J Mol Biol 2006; 359 : 848-62.

38. Bosch B, Blanco J, Pauls $\varepsilon$, et al. Inhibition of coreceptor-independent cell-to-cell human immunodeficiency virus type 1 transmission by a CD4-immunoglobulin G2 fusion protein. Antimicrob Agents Chemother $2005 ; 49:$ 4296-304.

39. Jolly C, Kashefi K, Hollinshead M, Sattentau QJ. HIV-1 cell to cell transfer across an Envinduced, actin-dependent synapse. J Exp Med 2004 ; 199 : 283-93.

40. Sol-Foulon N, Sourisseau M, Porrot F, et al. ZAP-70 kinase regulates HIV cell-to-cell spread and virological synapse formation. EMBO J 2007; 26:516-26.

41. Sherer NM, Lehmann MJ, Jimenez-Soto LF, et al. Retroviruses can establish filopodial bridges for efficient cell-to-cell transmission. Nat Cell Biol $2007 ; 9$ : 310-5.

42. Delamarre L, Rosenberg AR, Pique $C$, et al. A novel human T-leukemia virus type 1 cell-tocell transmission assay permits definition of SU glycoprotein amino acids important for infectivity. J Virol 1997; $71: 259-66$.
TIRÉS À PART

J.L. Darlix et D. Muriaux 